Multigrid Methods for Finite Elements 
Mathematics and Its Applications

Managing Editor:

M. HAZEWINKEL

Centre for Mathematics and Computer Science, Amsterdam, The Netherlands

Volume 318 


\section{Multigrid Methods for Finite Elements}

by

\section{V. Shaidurov}

Computing Center,

Russian Academy of Sciences,

Siberian Branch,

Krasnoyarsk, Russia

SPRINGER-SCIENCE+BUSINESS MEDIA, B.V. 
A C.I.P. Catalogue record for this book is available from the Library of Congress.

ISBN 978-90-481-4506-5 ISBN 978-94-015-8527-9 (eBook)

DOI 10.1007/978-94-015-8527-9

This is an updated and revised translation of the original work

Multigrid Methods of Finite Elements,

Moscow, Nauka @ 1989

Translated from Russian by N.B. Urusova

Printed on acid-free paper

All Rights Reserved

(C) 1995 Springer Science+Business Media Dordrecht

Originally published by Kluwer Academic Publishers in 1995

Softcover reprint of the hardcover 1st edition 1995

No part of the material protected by this copyright notice may be reproduced or utilized in any form or by any means, electronic or mechanical, including photocopying, recording or by any information storage and retrieval system, without written permission from the copyright owner. 


\section{Contents}

$\begin{array}{ll}\text { Preface } & \text { IX }\end{array}$

Introduction $\quad$ XI

1 Elliptic boundary-value problems and Bubnov-Galerkin method 1

1.1 Statements of differential problems and smoothness of solutions .... 1

1.1.1 Main notations of smoothness classes . . . . . . . . . . 2

1.1.2 Solving two abstract problems . . . . . . . . . . . 4

1.1.3 The Iirichlet problem for second-order elliptic equation . . . . 7

1.1.4 Boundary-value problems of third kind . . . . . . . . . 11

1.1.5 The Neumann problem . . . . . . . . . . . . . . . . 14

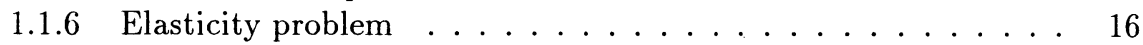

1.1.7 The Dirichlet problem for biharmonic equation . . . . . . . . 20

1.1.8 Boundary-value problems for plates . . . . . . . . . . 21

1.1.9 The mixed method for biharmonic equation . . . . . . . 23

1.1.10 The Stokes problem . . . . . . . . . . . . . . . 26

1.2 The general scheme of the Bubnov-Galerkin method . . . . . . . . . 29

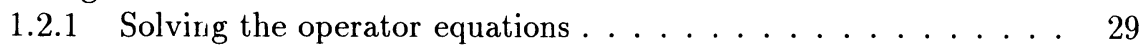

1.2 .2 The spectral problem . . . . . . . . . . . . . . . 31

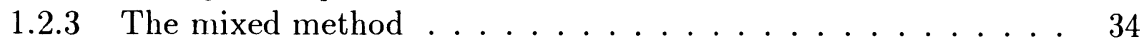

2 General properties of finite elements $\quad 37$

2.1 Classification of finite elements . . . . . . . . . . . . . . 38

2.2 Two-dimensional finite elements . . . . . . . . . . . . . . . . 39

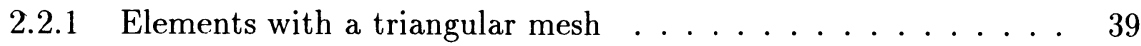

2.2 .2 Elements with a rectangular mesh . . . . . . . . . . . 42

2.3 Three-dimensional finite elements . . . . . . . . . . . . . . 45

2.3.1 Elements with a tetrahedral cell . . . . . . . . . . . . 47

2.3.2 Elements with a mesh shaped as a rectangular parallelepiped . . 49

2.4 Affine and isoparametric transformations . . . . . . . . . . 51

2.4.1 Curvilinear elements . . . . . . . . . . . . . . 51

2.4.2 Isopar ametric transformations of triangles . . . . . . . . 52

2.4.3 Isoparametric transformation of a square . . . . . . . . 55

2.5 Triangulation of two-dimensional domains $\ldots \ldots \ldots \ldots 7$ 
2.5.1 The boundary-correction algorithm . . . . . . . . . . 59

2.5.2 The depletion algorithm ............................. 60

2.5.3 The breaking algorithm .................... 61

2.6 Triangulation of the three-dimensional domains . . . . . . . . . 62

2.6.1 The boundary-correction algorithm .......... 63

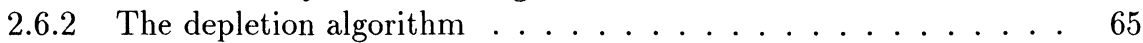

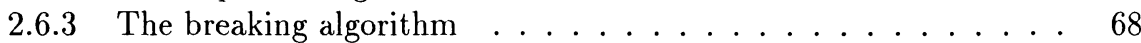

2.7 Other algorithms and refinement of triangulation . . . . . . . . 71

3 On the convergence of approximate solutions $\quad \mathbf{7 5}$

3.1 Interpolation by finite elements . . . . . . . . . . . . . . 76

3.1.1 Local interpolation . . . . . . . . . . . . . . . . . 76

3.1.2 Global interpolation ................. 78

3.1.3 The breaking algorithm for finite elements and nesting of basis

functions space ....................... 81

3.2 The convergence of the Bubnov-Galerkin method . . . . . . . . . 87

3.2.1 The solution of an operator equation . . . . . . . . . . 87

3.2.2 The spectral problem . . . . . . . . . . . . . . . 90

3.2 .3 The mixed method ................. 91

3.3 The use of quadrature formulae . . . . . . . . . . . . . . . 92

3.3.1 Influence of isoparametric or affine transformations . . . . . . 92

3.3.2 The ellipticity and the accuracy . . . . . . . . . . . . 93

3.3.3 The two-dimensional case ............... . . 95

3.3.4 The three-dimensional case . . . . . . . . . . . . . . 98

3.4 The approximation of boundary conditions . . . . . . . . . . . 100

3.4.1 A conformable triangulation and isoparametric elements . . . . 101

3.4.2 An unconformable triangulation and the penalty method . . . . 103

3.4.3 The mixed method ... . . . . . . . . . . . . 105

3.5 The Richardson extrapolation . . . . . . . . . . . . . 106

3.6 The conditionality of linear equations systems and diagonal . . . . . . . 112 normalization

4 General description of multigrid algorithms $\quad \mathbf{1 1 7}$

4.1 Solving the problems of parameters optimization . . . . . . . . . . . . . 118

4.2 An abstract setting the multigrid algorithms . . . . . . . . . . 125

4.2.1 The algebraic formulation of the multigrid algorithms . . . . . . 126

4.2.2 The multigrid operator of error suppression . . . . . . . . . . 127

4.3 The convergence in the symmetric variant . . . . . . . . . . . . . 129

4.3.1 The regular case .................. . . 129

4.3.2 The verification of the convergence criterion . . . . . . . . . . 132

4.3.3 The non-regular case . . . . . . . . . . . . . . . 135

4.3.4 The more regular case . . . . . . . . . . . . . . . . 139

4.4 A convergence for symmetric sign-indefinite operators . . . . . . . . 141

4.4.1 The convergence theorem ................ 141

4.4.2 Convergence in an ordinary situation ............. 144

4.5 General case. Convergence in initial norm . . . . . . . . . . . . . 146

4.5.1 Convergence criteria ............... 146 
4.5.2 A case of ordinary accuracy

4.6 The formulation of the convergence conditions for the Lagrange finite

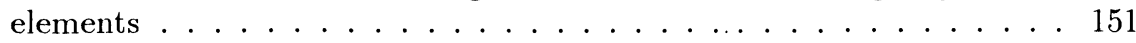

4.6.1 A symmetric positive-definite bilinear form . . . . . . . . . . 151

4.6.2 Symmetric sign-indefinite bilinear form . . . . . . . . . . . . 160

4.6.3 An asymmetric sign-indefinite bilinear form . . . . . . . . 161

4.7 Simultaneous application of the multigrid algorithms and the Richardson

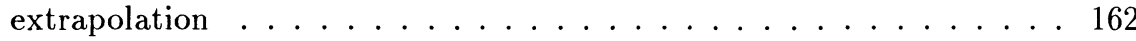

4.7.1 Convergence in energy norm .............. . . 164

4.7 .2 Convergence in $L_{2}$-norm . . . . . . . . . . . . . . . . 167

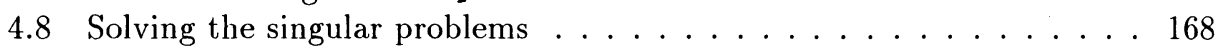

4.9. Solving the spectral problems . . . . . . . . . . . . . . 179

4.10 The algorithm in the symmetrized case . . . . . . . . . . . . . 192

4.10 .1 Convergence in $L_{2}$-norm . . . . . . . . . . . . . . . 192

4.10 .2 Convergence in "energy" norm . . . . . . . . . . . . . 198

4.11 The algorithm for the mixed method . . . . . . . . . . . . . . . . . . . . . . . . . . . . . . .

4.11 .1 Convergence in $L_{2}$-norm . . . . . . . . . . . . . . . . . . . 200

4.11.2 Convergence in "energy" norm . . . . . . . . . . . . 203

5 Realization of the algorithms for second-order equations 207

5.1 The two-dimensional Dirichlet problem . . . . . . . . . . 208

5.1.1 Properties of a differential and a difference problems . . . . . . 208

5.1.2 A calculation of the number of arithmetic operations . . . . . 210

5.1 .3 A numerical experiment . . . . . . . . . . . . . 214

5.1.4 Some statements for the other situations . . . . . . . . . . 215

5.1.5 Graphic representation of algorithms ........... 216

5.2 A modified algorithm for domains with a curvilinear boundary . . . . . 217

5.3 The problem with a pointwise singularity . . . . . . . . . . . . . 224

5.4 The three-dimensional Dirichlet problem . . . . . . . . . . . . 230

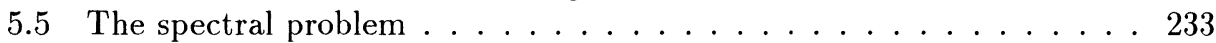

5.6 The boundary value problems of second and third kind . . . . . . . . 243

5.6.1 The third boundary value problem on a subordinate triangulation 244

5.6.2 The Neumann problem . . . . . . . . . . . . . 246

5.6.3 The third boundary value problem on regular grids . . . . . . 247

6 Solving nonlinear problems and systems of equations 251

6.1 Nonlinear problems with an operator of monotone type . . . . . . . 251

6.2 Solution of quasi-linear equations of order $2 \ldots \ldots . \ldots 255$

6.2.1 The equation with a weak nonlinearity . . . . . . . . . 255

6.2.2 The Newton method and the multigrid algorithm . . . . . . . 259

6.2.3 The magnetostatic problem ................... 264

6.2.4 The simplest two-stage iterative process . . . . . . . . . . 267

6.2 .5 Other linearizations . . . . . . . . . . . . . . . 270

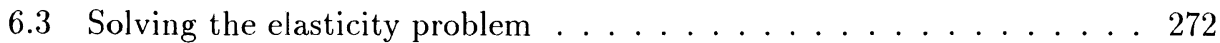

6.3.1 The elasticity plane problem . . . . . . . . . . . . 272

6.3.2 Projective-difference problem ............ 273 
6.3.3 The multigrid iterative algorithm . . . . . . . . . . 274

6.3 .4 The numerical experiment . . . . . . . . . . . . . . 274

6.3.5 Possible generalizations . . . . . . . . . . . . . . 275

6.4 The biharmonic equation . . . . . . . . . . . . . 276

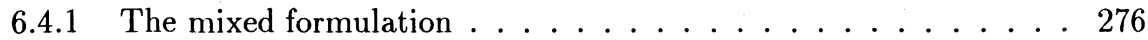

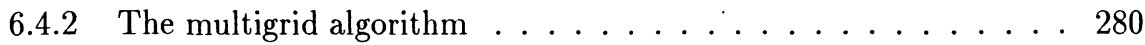

6.4 .3 Another approach . . . . . . . . . . . . . . 286

6.5 The stationary Stokes problem . . . . . . . . . . . . . . . . . 288

6.5.1 The formulation of the differential problem . . . . . . . . 288

6.5.2 The discrete problem . . . . . . . . . . . . . . . . 291

6.5 .3 The multigrid algorithm $\ldots \ldots \ldots \ldots \ldots \ldots$

6.5.4 Other combinations of finite elements . . . . . . . . . . 300

6.6 The stationary Navier-Stokes problem . . . . . . . . . . . . . . 301

6.6.1 The formulation of a differential problem . . . . . . . . . 302

6.6 .2 The discrete problem . . . . . . . . . . . . . . 303

6.6.3 The Newton method and the multigrid algorithm . . . . . . 303

6.6 .4 The main result . . . . . . . . . . . . . . 307

Bibliography $\quad 313$

$\begin{array}{ll}\text { Subject index } & 327\end{array}$ 


\section{Preface}

At present, the finite element method has become one of the most widespread and effective methods to solve a variety of problems in mathematical physics and engineering. Its popularity is connected with universality and simplicity of mathematical form for a wide range of problems, in combination with flexibility of numerical algorithms, enabling concrete properties of an individual problem to be considered. To no small degree, its success has been due to the development of high-speed computer techniques and by advances in the fields of projective methods and approximation theory.

The material for this book is based on a series of works of various mathematiciants, especially the monographs by P.G.Ciarlet, S.F.McCormick, L.A.Oganesyan and L.A.Rukhovets, G.I.Marchuk and V.I.Agoshkhov, G.Fix and G.Streng and W.Hackbusch, as well as on results of research carried out by the author at the Krasnoyarsk Computing Center of the Siberian Division of the Russian Academy of Sciences.

Traditional mathematical principles of the finite element method will be discussed briefly enough in view of the large number of monographs on the subject. A more detailed description is given of important technical problems which are not treated well in the literature, namely: triangulation techniques of two- and three-dimensional domains, efficient quadrature formulae, and using a mixed method as a systematic way to change burdensome main conditions in basis subspaces for natural ones. Such a substitution allows, for example, to simplify a treatment of the Dirichlet non-uniform boundary conditions, to reduce the biharmonic equation to a system of second-order equations and to remove the rather inconvenient requirement of solenoidality of basis functions in the Stokes and Navier-Stokes problems.

When considering specific problems, the simplest and most widespread finite elements were used, at the same time noting ways of using more complicated ones.

The greatest attention is paid to the numerical solution of algebraic problems with large sparse matrices following discretization by means of multigrid algorithms, first proposed by R.P.Fedorenko in 1961. These algorithms lead to a wide range of applications and, at the same time, such an unimproved property as a finite number of arithmetical operations per one unknown for achievment of the accuracy stipulated by the convergence order.

Hundreds of present publications reveal that the practical use of these methods are often ahead in efficiency of other direct and iterative methods.

This book presents a more detailed study of multigrid methods applied to concrete problems. It also includes some new results on the optimization of iterative parameters, solving generalized spectral problems, applying a mixed finite element method, and solving problems with peculiarities and curvilinear boundaries.

This book is intended for engineers and research workers using or developing the finite element method for solving practical problems. The material is discussed in such a way that the main parts may be applied, directly or by close analogy, to many more problems. The relatively self-contained and detailed discussion is intelligible to senior-year students of mathematics and engineering specialities.

The style and the material of the book underwent essential changes in comparison with Russian variant published in 1989. Discussions with collegues, new results of other scientists influenced the description. Besides, some proofs were specified and 
inaccuracies were corrected.

This work became possible due to a partial financial support of Mathematical Department of the Magdeburg Otto-von-Guericke University, Berlin Institute of Applied Analysis and Stochastics, Beijing Institute of Systems Science. The auther acknoleges them for the possibility to take part in several conferences, to work in excellent libraries, to discuss with collegues from many countries. The author is deeply grateful to G.I.Marchuk and other scientists including N.S.Bakhvalov, R.P.Fedorenko, L.Tobiska, V.G.Korneev, Q.Lin, E.G.D'jakonov, U.Langer, whose advice and remarks have led to improvement. The author also thanks S.N.Bogdanov, A.V.Smirnov for numerical experiments carried out and T.G.Belokopytova, N.B.Urusova, S.V.Belokopytov, J.Fuhrmann, S.Hengst for the help during the work at the manuscript.

V.Shaidurov 


\section{Introduction}

The history of the finite element method goes back to the works of V.Ritz, I.G.Bubnov and B.G.Galerkin. The constructive foundation of the method is a variational form of the problem and the use of splines with small supports called finite elements. Applying this method to a differential problem gives a finite-dimensional system of linear (or non-linear) algebraic equations, the solution of which yields parameters of splines approximating a sought function.

Solving a concrete problem of mathematical physics by using this method usually involves 4 main stages:

1. a variational formulation of the problem;

2. a choice of finite elements and a triangulation of the geometrical domain, i.e., dividing the domain into small supports of finite elements of a given form;

3. an assembly of the system of algebraic equations, often by using quadrature formulae;

4. solving the algebraic equations obtained.

In spite of the fact that there exists a great number of books on the finite element method, when this method is constantly being developed, questions arise at each of these stages indicated above which are not discussed well in the literature. For the first stage, a mixed variational formulation becomes lately a means of greater importance. Its application permits to use simpler finite elements, to raise the accuracy and efficiency of the numerical realization of problems such as the biharmonic equation, and the Stokes and Navier-Stokes problems. As far as the second stage is concerned, there is no a systematic discussion of the automatic triangulation of geometrical domains, especially of three-dimensional ones. The requirement of the third stage for calculations to be efficient puts quadrature formulae at the forefront, which, as a rule, are little-used for calculations. Finally, the fourth stage is not in practice discussed in the literature on finite elements. This is due to the fact that systems solved by means of a great number of direct and iterative methods. The monograph by A.A.Samarski and E.S.Nikolaev [156] provides a good overview of these methods.

One of the most effective and rather universal iterative methods for solving the systems obtained has become lately the multigrid method. Two approaches form its algorithmic base. We can illustrate these by an example of a grid problem corresponding to a rather fine triangulation and having a large number of unknowns. The first approach consists in consecutively solving the grid problems similar to an initial problem but corresponding to coarser triangulations. Solving begins on the most coarse triangulation, where it can be made efficiently. Then the obtained solution is interpolated on a finer triangulation and used as an initial approximation having an accuracy close to a satisfactory one. Thus, much fewer iterations in the iterative process are needed to obtain the required accuracy. This approach was widely practised in the times of manual and mechanized calculations when one uses all the costly information obtained from the coarse grid if he was forced to pass on to a finer grid. The second approach was suggested by R.P.Fedorenko in his article [53]. It is based on a fast convergence of some iterative processes for higher harmonics, which permits to decrease 
their contribution into the error essentially within a few iterations. Lower harmonics converge far slower and so they make up the most part of the error. As a result, the error becomes some smoothly changing grid function for which a system of grid equations can be written with residual on the right-hand side. In principle, this system can be solved, and the error can be found, but such a way is rather expensive. Instead of this note that a differential problem with a sufficiently smooth solution can be brought in correspondence to this system. For this differential problem, we again construct a grid on a coarser triangulation (for example, two or three times coarser). Such a system can be solved more efficiently since the number of unknowns and equations, for example, in the two-dimensional case, is four or nine times less. By virtue of stated smoothness, its solution approximates the sought error on this triangulation well enough.

Now interpolate it (even if linearly) onto a finer triangulation. In view of smoothly changing the error will be found with a rather high accuracy. Further one can construct some more iterations to suppress the high-frequency component following an interpolation or repeat once again all the procedure of transition to a coarser triangulation. Solving a grid problem on a triangulation twice or three times coarser may be still expensive, so it also can be solved approximately by using the described technique of the transition to a coarser triangulation. This method for decreasing the dimension can be used until going on to the coarsest triangulation on which the solution can be obtained easily enough by using the direct method.

Though the multigrid method is relatively complicated in the algorithmic sense, in 1964 R.P.Fedorenko [54] managed to prove its convergence for the finite-difference analogue of Poisson equation on square.

In 1966 N.S.Bakhvalov [19] proved this method to be optimal in respect of the number of arithmetical operations needed to achieve the accuracy agreed with the convergence order.

According to asymptotical estimates of efficiency, the method was ahead of other known algorithms but for a time its quality was veiled in its complicated logic and hard mathematical substantiation.

At a certain stage of developing the finite element method, a new mathematical apparatus and program realizations sufficiently decreased expense of the algorithm and simplified its validating. That is why at the end of 70 th an increase has begun and is still in progress in the amount of the literature on multigrid methods.

The monograph on the convergence theory of multigrid methods by W.Hackbush [68], owing to an abundance of the results accumulated and presented, should be defined as a reference book sooner. Therefore, when writing this book one of the purposes was to discuss systematically and to study in detail this promising method to be applied to the most frequently-used boundary problems of mathematical physics.

The structure and the substantiation of the multigrid method for finite elements are closely connected with the first three stages of discretization. This requires the material to be discussed in strict sequence.

Chapter 1 presents typical linear boundary problems of order 2 or 4 , explanes how to pass from a classical operator formulation to a generalized variation formulation including the mixed one. For the discussion to be uniform, only one of the variation principles was considered, namely, the Bubnov-Galerkin method. Most of the results can be applied to Ritz method as well as to the method of least squares. 
Chapter 2 treats general properties of finite elements and has a reference nature on the simplest and frequently-used finite elements as well as triangulation techniques of the two- and three-dimensional regions including a condensation of the triangulation near peculiar points and lines.

Chapter 3 deals with the convergence of the approximate solutions of the BubnovGalerkin method in the following sequence: approximation properties of finite elements, convergence conditions of approximate solutions, a choice of efficient quadrature formulae, approximation techniques for boundary and for main boundary conditions, and raising the accuracy of approximate solutions in terms of Richardson's extrapolation from different grids.

Chapter 4 gives a description and a substantiation of the convergence of several multigrid method modifications on a sequence of nested triangulations. The algorithms are given in the recurrent form with respect to different situations. At first, convergence conditions are discussed in terms of the abstract algebraic interpretation. It gives us a possibility to implement the theory to both the finite difference and the finite element methods. Then we specify this theory in terms of the finite element method. Therefore the multigrid methods have been classified with respect to the properties of the initial variational problems: for self-ajoint problem, non-self-ajoint one, spectral problem, mixed formulation, and so on.

The chapter also gives solutions of auxiliary problems for an optimization of iterative parameters. They permit a more speedly convergence of iterative algorithms in different norms. In the following sections this is confirmed by some numerical results.

Chapter 5 treats realizations of multigrid algorithms for elliptic equations of order 2. First, by an example of the two-dimensional Dirichlet problem a comparison is given between variant algorithms and among them have been chosen the optimal ones in respect of the number of arithmetical operations. Numerical experiments are given to illustrate these results.

A technique of constructing the nested grids for problems in a domain with curvilinear boundary has been considered as well as concrete realizations of iterative algorithms based on this technique.

Realizations of iterative algorithms have been discussed for the boundary problem with a peculiarity in the angle, when triangulation is locally condensed, as well as for the three-dimensional Dirichlet problem, the second and third boundary problem. Studying the three-dimensional problem does not show an essential distinction between it and the two-dimensional one in realization of multigrid algorithms as well as their substantiation and estimation of efficiency.

Also the spectral problem, it gives the generalized algebraic spectral problem $A u=$ $\lambda B u$ with symmetric non-diagonal matrices. The algorithm constructed on a sequence of triangulations permits to find an individual eigenvalue and corresponding eigenfunctions or groups of such functions in case of multiple eigenvalue, the previous eigenvalue and eigenfunctions being not calculated.

Chapter 6 explains, by concrete examples, how to generalize the results, so that they could be applied to non-linear equations and systems. In the first two sections, general results on the convergence of finite elements for non-linear problems with monotonetype operators have been discussed, as well as two typical non-linear problems applied in appendices have been solved by using multigrid algorithms. The solution of the pla- 
nar elasticity problem shows possibility of generalizing the constructed algorithms and of their applying to elliptic systems. Among many well-known discretization methods of biharmonic equation has been considered a mixed formulation of the finite element method resulting in a system of two Poisson equations with coupled boundary conditions. The generalized formulation contains only first derivatives and so it is not necessary to use complex basis functions from the class $C^{1}(\Omega)$. The mixed formulation has also been used to discretize the stationary Stokes and Navier-Stokes problems. Here we used the simplest combination of the finite elements, linear elements for velocities and constant elements for pressure.

In the three last problems, the matrices of algebraic systems obtained have a spectrum with alternating signs, which is typical for the mixed formulation of the finite element method. However, the application of multigrid algorithms to them is as efficient as to positive-definite matrices.

A few words should be said about the discussing form. Formulae, theorems have two-digit numeration but lemmata, remarks have three-digit one own in each chapter. In the three last chapters, as it may seem, the material is discussed rather monotonously, especially when formulating stages of discretization and multigrid algorithms for algebraic equations obtained. It is due to brief repetitions of the most important points for the reader interested in solving a certain boundary problem to obtain the necessary information on studying a small amount of the preceding material. 